\title{
Horizontal Stenting via Retrograde Route for Recurrent Ruptured Posterior Communicating Artery Aneurysm after Clipping: A Case Report and Literature Review
}

\author{
Michiyasu Fuga ${ }^{1}$, Toshihide Tanaka ${ }^{1}$, Rintaro Tachi ${ }^{1}$, Ryo Nogami $^{1}$, Akihiko Teshigawara ${ }^{1}$, \\ Toshihiro Ishibashi $^{2}$, Yuzuru Hasegawa ${ }^{1}$, and Yuiichi Murayama ${ }^{2}$ \\ ${ }^{1}$ Tokyo Jikei University Kashiwa Hospital \\ ${ }^{2}$ Jikei University School of Medicine
}

October 28, 2021

\begin{abstract}
Treatment of recurrent ruptured aneurysms incorporating a branch vessel arising from the dome is challenging. Here, we attempted horizontal stent-assisted coil embolisation via a retrograde route from the contralateral internal carotid artery to treat a small ruptured posterior communicating artery aneurysm incorporating a foetal variant posterior cerebral artery after clipping.
\end{abstract}

Horizontal Stenting via Retrograde Route for Recurrent Ruptured Posterior Communicating Artery Aneurysm after Clipping: A Case Report and Literature Review

Michiyasu Fuga, M.D. ${ }^{1}$, Toshihide Tanaka, Ph.D. ${ }^{1}$, Rintaro Tachi, M.D. ${ }^{1}$, Ryo Nogami, M.D. ${ }^{1}$, Akihiko Teshigawara, M.D. ${ }^{1}$, Toshihiro Ishibashi, M.D. ${ }^{2}$, Yuzuru Hasegawa, M.D., D.M.Sc. ${ }^{1}$, Yuichi Murayama, M.D., D.M.Sc. ${ }^{2}$

${ }^{1}$ Department of Neurosurgery, Jikei University School of Medicine, Kashiwa Hospital, Chiba, Japan ${ }^{2}$ Department of Neurosurgery, Jikei University School of Medicine, Tokyo, Japan

Correspondence: Michiyasu Fuga, M.D.

Department of Neurosurgery, Jikei University School of Medicine, Kashiwa Hospital

163-1 Kashiwashita, Kashiwa-shi, Chiba 277-8567, Japan

Tel: +81-4-7164-1111, Fax: +81-4-7163-3488, E-mail: fugamichiyasu@icloud.com

\section{Abstract}

Treatment of recurrent ruptured aneurysms incorporating a branch vessel arising from the dome is challenging. Here, we attempted horizontal stent-assisted coil embolisation via a retrograde route from the contralateral internal carotid artery to treat a small ruptured posterior communicating artery aneurysm incorporating a foetal variant posterior cerebral artery after clipping.

\section{KEYWORDS}

acutely angled; anterior communicating artery; foetal variant posterior cerebral artery; recurrent aneurysm; stent assisted

\section{Abbreviations and Acronyms}


AcomA: anterior communicating artery

A1: horizontal segment of the anterior cerebral artery

BAT: balloon-assisted technique

CTA: computed tomography angiography

DCT: double-catheter technique

ICA: internal carotid artery

ICAG: internal carotid angiography

PCA: posterior cerebral artery

PcomA: posterior communicating artery

SAC: stent-assisted coiling

\section{INTRODUCTION}

Surgical treatment for recurrent aneurysms after clipping is considered cumbersome, especially for reclipping because of existing clips, scar tissue and severe adhesions. ${ }^{1,2}$ Therefore, to avoid additional surgical insult, coil embolisation is considered advantageous compared with craniotomy surgery. ${ }^{3,4}$ However, endovascular surgery for recurrent aneurysm incorporating a branch vessel arising from the dome is quite difficult. Stentassisted coiling (SAC) could overcome such difficulties compared with the standard technique. Various stentassisted techniques have been applied for SAC of bifurcated aneurysms. ${ }^{5-7}$ Horizontal stenting involves placement of the stent across the aneurysm neck parallel to the bifurcated branch and axis of the neck, achieving optimal neck formation with only one stent. ${ }^{8}$ This procedure reduces the risk of thromboembolic complications and medical costs. ${ }^{9}$ In cases of stent deployment for a branch vessel acutely angled from the aneurysm sac, a retrograde approach via the anterior communicating artery (AcomA) allows easier guidance of the microcatheter than the anterograde approach, ${ }^{9-11}$ especially for retreatment after clipping. The technical limitations of this approach depend on the AcomA or posterior communicating artery (PcomA) diameter. The NeuroForm Atlas stent (Stryker, Kalamazoo, MI, USA) enables guidance to smaller blood vessels, and is easier than guidance via microcatheter since the outer diameter in only $1.7 \mathrm{~F}$. Here, we describe a case of ruptured small PcomA aneurysm incorporating a foetal variant posterior cerebral artery (PCA) after clipping, treated by horizontal stenting using a NeuroForm Atlas stent delivered via AcomA from the contralateral internal carotid artery (ICA).

\section{CASE PRESENTATION}

An 80-year-old woman who had underwent clipping surgery for a ruptured PcomA aneurysm 10 years earlier at another hospital developed subarachnoid haemorrhage (Hunt \& Hess grade 2; Fisher group 3). Computed tomography angiography (CTA) and 3-dimensional digital subtraction angiography demonstrated a residual PcomA aneurysm with a 2.7-mm wide-necked aneurysm just proximal to the clip and an isolated foetal variant PCA originating from the aneurysm sac (Fig. 1a, b). Based on these findings, rupture of a residual PcomA aneurysm was diagnosed.

\section{Treatment and Technique}

Because the residual aneurysm was small with a wide neck, and the foetal variant PCA branch vessel was arising from the dome, SAC was selected to preserve the PcomA. The stent was used with the consent of the patient and their family due to the off-label use for ruptured aneurysm in Japan. Dual antiplatelet agents (loading doses: $200 \mathrm{mg}$ aspirin, $200 \mathrm{mg}$ cilostazol) were administered continuously before and after embolisation. Endovascular treatment was performed under general anaesthesia and heparin was administered systemically as an initial 3000-U bolus, followed by $1000 \mathrm{U} / \mathrm{h}$ with monitoring of whole-blood activated clotting time throughout the procedure. An 8-Fr FlowGate2 balloon guide catheter (Stryker) was guided to the petrous segment of the right ICA via the right femoral artery. An Excelsior SL-10 microcatheter 
(Stryker) was guided into the aneurysm from the 8-Fr FlowGate2 placed in the right ICA. After that, a Target 360 Nano coil (Stryker) was inserted into the aneurysm. However, because the aneurysm was small and wide-necked, and the foetal variant PCA branch vessel was arising from the dome, placing the coil into the aneurysm alone so as to avoid herniating the ICA trunk and PcomA was difficult. The PcomA branched at an acute angle $\left(40^{*}\right)$ (Fig. 1a, b) and the orifice of the PcomA was not well visualised due to the existing clip (Fig. 1c). The stent delivery microcatheter thus could not be guided in an anterograde manner to the PcomA from the ipsilateral ICA. A 5-Fr Envoy (Codman Neurovascular, Raynham, MA, USA) was guided to the cervical segment of the contralateral ICA via the left femoral artery. Because the AcomA was 1.1 $\mathrm{mm}$ in size (Fig. 2a), the stent delivery catheter was navigated in a retrograde manner to the contralateral horizontal segment of the anterior cerebral artery (A1) (Fig. 2b), ipsilateral terminal ICA (Fig. 2c), and right foetal variant PCA (Fig. 2d) from the contralateral ICA via the AcomA. In the process of guiding the catheter from the contralateral ICA to the ipsilateral ICA across the AcomA, the microcatheter was mistakenly placed into the artery of Heubner. After guiding the stent delivery catheter to the right foetal variant PCA, a microcatheter for coil delivery via the 8-Fr FlowGate2 placed at the ipsilateral ICA was advanced into the aneurysm sac. The self-expandable $4.0 \mathrm{~mm} \times 21 \mathrm{~mm}$ NeuroForm Atlas stent was navigated thru the Excelsior SL-10 placed in the right PcomA and deployed from the right PcomA into the terminal right ICA (Fig. 3a). The coil delivery microcatheter was gaoled. The aneurysm was completely occluded by three Target 360 Nano coils using the gaoled microcatheter (Fig. 3b). Final right internal carotid angiography (ICAG) demonstrated complete occlusion of the aneurysm by appropriate placement of the stent with preservation of the parent arteries, including the terminal right ICA and PcomA. After embolisation, the patient suffered left hemiparesis. Postoperative magnetic resonance imaging showed cerebral infarction in the caudate head and internal capsule consistent with the territory of the right artery of Heubner. Dual antiplatelet therapy has been continued for three months. The patient was transferred to a rehabilitation hospital with a modified Rankin scale score of 4 as of two months after endovascular treatment. Follow-up right ICAG two years after embolisation demonstrated persistent complete occlusion without recanalisation (Fig. 3c).

\section{DISCUSSION}

The difficulties and pitfalls in the present case primarily involved the small, recurrent aneurysm. Reclipping for recurrent aneurysms following surgical clipping tends to be cumbersome, because the direction of insertion for the clip blade is restricted by existing clips, scar tissue and adhesions. ${ }^{1,2}$ Rabinstein et al. reported on 21 postsurgical neck remnants of aneurysms treated by endovascular coiling without major complications. ${ }^{3}$ Subarachnoid haemorrhage and symptomatic aneurysmal regrowth were not observed after endovascular retreatment during follow-up (mean, 22 months). ${ }^{3}$ For these reasons, coil embolisation is more advantageous for recurrent aneurysm following clipping. ${ }^{4}$

In endovascular treatment, if the aneurysm is small, has a wide neck, and normal branch vessels arising from the dome, treatment with a simple technique is difficult. Some adjunctive techniques are required, such as balloon-assisted technique (BAT), double catheter technique (DCT), and SAC. Abdalkader et al. pointed out that the balloon deflation phase during BAT for small aneurysms might increase the risk of coil protrusion and coil migration. ${ }^{1,2}$ When DCT is applied for small and ruptured aneurysms, placing two microcatheters in the aneurysm sac while avoiding perforation is difficult. ${ }^{13}$ Based on such description, SAC is considered a more appropriate treatment. Two options are available for SAC to treat bifurcation aneurysms, using the antegrade or retrograde route. Y-stenting is useful for access via the antegrade route. ${ }^{5,14}$ However, Y-stenting requires two stents, which might increase the risk of thrombotic complications. ${ }^{815}$ Johnson et al. pointed out that Y-stenting represents an independent risk factor for permanent disabling neurological complications. ${ }^{16}$

The technical difficulties in this case involved the acute angle between the parent artery and PcomA. Guiding the stent delivery catheter into a branched vessel with an acute angle is difficult. Moreover, the existing clip obscures the PcomA/ICA junction in cases of recurrent aneurysm after surgical clipping. Anterograde guidance of the microcatheter into the branch vessel acutely angled from the aneurysm sac, particularly one with an existing clip, seems disadvantageous, therefore a retrograde approach should be assumed in advance. The retrograde approach facilitates guidance of the microcatheter toward the target vessel, because the angle 
of bifurcation between the terminal ICA and PcomA becomes obtuse. In addition, this procedure facilitates horizontal stenting, which covers the neck horizontally along the wall of the efferent and afferent arteries with a single stent. This technique not only leads to tight packing to reduce recanalisation, but also prevents thrombotic complications compared with Y-stents.

According to previous reports, 15 aneurysms, including that in the present case, have been treated by horizontal stenting delivered in a retrograde manner via the AcomA from the contralateral ICA. ${ }^{7,9,10,17-22}$ As shown in Table, all PcomA aneurysms incorporated a foetal variant PCA. The aneurysm type was unruptured in 11 cases and ruptured in 3, including 4 recurrent aneurysms. No aneurysm recurrence was seen after previous clipping, except in the present study. Most aneurysms were [?] $\mathrm{mm}$ in size and had a wide neck. Ahmed et al. reported that in the treatment of a ruptured 2-mm carotid terminus/A1 aneurysm, a double Enterprize stent (Codman Neurovascular) construct was deployed horizontally across the aneurysm in "telescopic" fashion for inflow coverage. ${ }^{21}$

The angle of bifurcation between the ICA and PcomA is a crucial factor in successful stent deployment. The bifurcated vessel angle toward the anterograde flow between the afferent and efferent arteries where the stent is placed might be important for efficient and safe catheter delivery. The angle of bifurcation between the ICA and PcomA was acute in all cases (mean, 48deg; range, 40-53deg). The angle was the steepest in the present case, at 40deg. Calibre of the AcomA is another important factor for the retrograde approach. To ensure success of horizontal stenting via the retrograde route, the calibre of the AcomA should be examined in advance. The minimum size of the AcomA was $0.9 \mathrm{~mm}$ (range, 0.9-1.9 mm) in the cases we identified. A Prowler Select Plus (Codman Neuroendovascular, Johnson \& Johnson, Miami, FL, USA) was used as a stent delivery catheter in most cases, with a smaller-diameter (outer diameter: $1.7 \mathrm{~F}$ ) catheter (Headway 17; MicroVention, Tustin, CA, USA and Excelsior SL-10) used in only two cases. Kim et al. pointed out that a communicating artery diameter $>1 \mathrm{~mm}$ is needed to navigate the stent delivery catheter. ${ }^{17}$ In the present case, AcomA diameter was $1.1 \mathrm{~mm}$ on CTA, consequently the microcatheter was easily delivered to the PcomA. Note that a careful procedure is needed to prevent guidewires and microcatheters from migrating into perforating arteries, including the artery of Heubner, which runs parallel to A1. At that time, an accurate roadmap is essential to avoid complications when coil embolisation is performed via a retrograde route from the AcomA.

\section{CONCLUSION}

Endovascular treatment of a recurrent PcomA aneurysm incorporating an acutely angled foetal variant PCA after clipping is very difficult. The retrograde approach using a NeuroForm Atlas stent is one effective option for coil embolisation for aneurysms with a steep ICA-PcomA angle.

\section{ACKNOWLEDGMENTS}

None

\section{CONFLICTS OF INTEREST}

The authors report no conflict of interest.

\section{AUTHOR CONTRIBUTIONS}

MF, TT, TI, YH, and YM created the research question for this paper. RT, RN, and AT took part in the data collection process. MF wrote the final document. All authors gave substantial contributions to the conception or design of the work, drafted it, and critically revised it for important intellectual content. All authors reviewed and approved the final manuscript.

\section{ETHICAL APPROVAL}

Not applicable.

\section{FUNDING}


This work did not receive any grants from funding agencies in the public, commercial, or not-for-profit sectors.

\section{REFERENCES}

1. Drake CG, Friedman AH, Peerless SJ. 1984. Failed aneurysm surgery. Reoperation in 115 cases. $J$ Neurosurg . 61(5):848-856.

2. Giannotta SL, Litofsky NS. 1995. Reoperative management of intracranial aneurysms. J Neurosurg . 83(3):387-393.

3. Rabinstein AA, Nichols DA. 2002. Endovascular coil embolization of cerebral aneurysm remnants after incomplete surgical obliteration. Stroke . 33(7):1809-1815.

4. Forsting M, Albert FK, Jansen O, et al. 1996. Coil placement after clipping: endovascular treatment of incompletely clipped cerebral aneurysms. Report of two cases. J Neurosurg . 85(5):966-969.

5. Chow MM, Woo HH, Masaryk TJ, Rasmussen PA. 2004. A novel endovascular treatment of a widenecked basilar apex aneurysm by using a Y-configuration, double-stent technique. AJNR Am J Neuroradiol . 25(3):509-512.

6. Akpek S, Morsi H, Benndorf G, Strother CM, Mawad ME. 2004. Reconstruction of the basilar tip with T stent configuration for treatment of a wide-neck aneurysm. J Vasc Interv Radiol . 15(9):1024-1026.

7. Benndorf G, Klucznik RP, Meyer D, Strother CM, Mawad ME. 2006. "Cross-over" technique for horizontal stenting of an internal carotid bifurcation aneurysm using a new self-expandable stent: technical case report. Neurosurgery . 58(1 Suppl):ONS-E172; discussion ONS-E172.

8. Cross DT, 3rd, Moran CJ, Derdeyn CP, Mazumdar A, Rivet D, Chicoine MM. 2005. Neuroform stent deployment for treatment of a basilar tip aneurysm via a posterior communicating artery route. AJNR Am $J$ Neuroradiol . 26(10):2578-2581.

9. Albuquerque FC, Gonzalez LF, Hu YC, Newman CB, McDougall CG. 2011. Transcirculation endovascular treatment of complex cerebral aneurysms: technical considerations and preliminary results. Neurosurgery . 68(3):820-829; discussion 829-830.

10. Kwon HJ, Cho YD, Lim JW, et al. 2019. Retrograde stenting through anterior communicating artery in coil embolization of the posterior communicating artery aneurysm: Contralateral approach. J Neuroradiol .

11. Fitzpatrick D, Chen M, Meyers PM. 2006. Horizontal Neuroform stent deployment for a ruptured basilar terminus aneurysm via the posterior communicating artery. J Vasc Interv Radiol . 17(10):1687-1691.

12. Abdalkader M, Piotin M, Chen M, et al. 2020. Coil migration during or after endovascular coiling of cerebral aneurysms. J Neurointerv Surg . 12(5):505-511.

13. Cloft HJ, Kallmes DF. 2002. Cerebral aneurysm perforations complicating therapy with Guglielmi detachable coils: a meta-analysis. AJNR Am J Neuroradiol . 23(10):1706-1709.

14. Thorell WE, Chow MM, Woo HH, Masaryk TJ, Rasmussen PA. 2005. Y-configured dual intracranial stent-assisted coil embolization for the treatment of wide-necked basilar tip aneurysms. Neurosurgery . 56(5):1035-1040; discussion 1035-1040.

15. Pride GL, Jr., Welch B, Novakovic R, et al. 2009. Retrograde crossing stent placement strategies at the basilar apex for the treatment of wide necked aneurysms: reconstructive and deconstructive opportunities. J Neurointerv Surg . 1(2):132-135.

16. Johnson AK, Munich SA, Tan LA, Heiferman DM, Keigher KM, Lopes DK. 2015. Complication analysis in nitinol stent-assisted embolization of 486 intracranial aneurysms. J Neurosurg . 123(2):453-459. 
17. Kim YJ, Ko JH. 2015. Coiling of a recurrent broad-necked posterior communicating aneurysm incorporating a fetal cerebral artery: A technical case report. Interv Neuroradiol . 21(1):44-49.

18. Kelly ME, Turner R, Gonugunta V, et al. 2007. Stent reconstruction of wide-necked aneurysms across the circle of Willis.Neurosurgery . 61(5 Suppl 2):249-254; discussion 254-245.

19. Siddiqui MA, J JB, Lindsay KW, Jenkins S. 2009. Horizontal stent-assisted coil embolisation of widenecked intracranial aneurysms with the Enterprise stent-a case series with early angiographic follow-up. Neuroradiology . 51(6):411-418.

20. Puri AS, Erdem E. 2009. Unusual Intracranial Stent Navigation through the Circle of Willis in a Patient with Recurrent Basilar Tip Aneurysm during Stent-Assisted Coiling. A Case Report. Interv Neuroradiol . 15(1):81-86.

21. Ahmed ME, Lum C, Lesiuk H, Iancu D, dos Santos M. 2014. Navigation of Stents across Communicating Arteries for Aneurysm Embolization. Can J Neurol Sci . 41(2):193-199.

22. Kitahara T, Hatano T, Hayase M, Hattori E, Miyakoshi A, Nakamura T. 2017. Jailed doublemicrocatheter technique following horizontal stenting for coil embolization of intracranial wide-necked bifurcation aneurysms: A technical report of two cases. Interv Neuroradiol . 23(2):117-122.

Table.

Horizontal stenting by retrograde technique via anterior communicating artery

Figure legends

\section{Figure 1}

Axial computed tomography angiography source images (a) and 3-dimensional digital subtraction angiography (image from directly above) (b) demonstrating a $2.7-\mathrm{mm}$ broad-necked posterior communicating artery (PcomA) aneurysm residuum (A) just proximal to the clip incorporating an acute angled foetal posterior cerebral artery. These images show an acute angle $\left(40^{\circ}\right)$ subtended by the PcomA (white arrowhead) and proximal internal carotid artery (white arrow). The orifice of the PcomA is not well visualised on right internal carotid artery angiography (c) due to the existing clip.

\section{Figure 2}

Axial computed tomography angiography source images (a) show anterior communicating artery with a diameter of $1.1 \mathrm{~mm}$. Left internal carotid artery (ICA) angiography (b) under balloon inflation in the right proximal ICA and the fluoroscopic view (c, d) show retrograde navigation of the stent delivery catheter to the contralateral horizontal segment of the anterior cerebral artery (b), ipsilateral terminal ICA (c) and right foetal posterior cerebral artery (d) from the contralateral ICA.

\section{Figure 3}

a) Fluoroscopic view shows the NeuroForm Atlas stent deployed from the right foetal posterior cerebral artery to the terminal right internal carotid artery (ICA).

b) Right ICA angiography shows complete occlusion of the aneurysm without coil migration into the parent arteries.

c) Right ICA angiography at the two-year follow-up after endovascular treatment demonstrates complete occlusion without recanalisation or in-stent stenosis. 

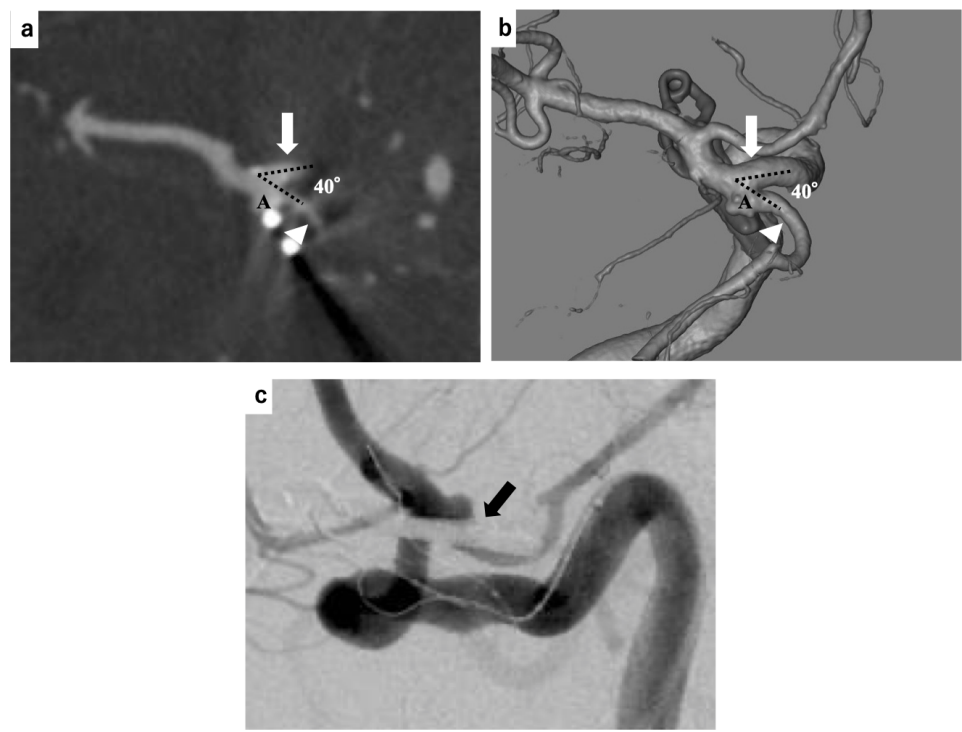

Figure 1.

Figure 2.
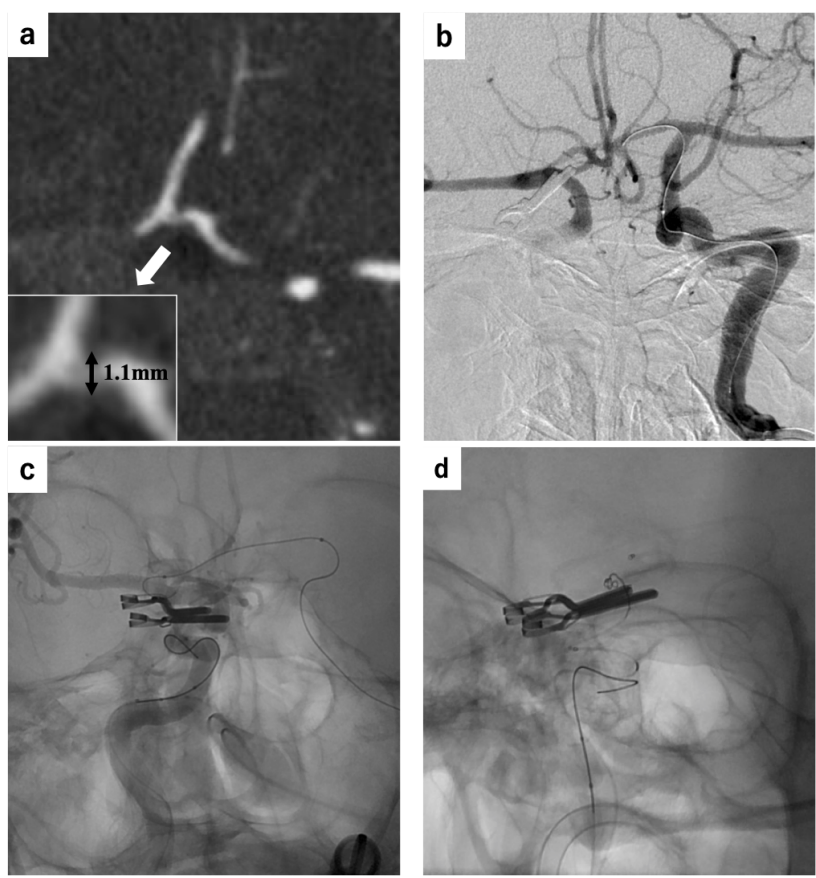

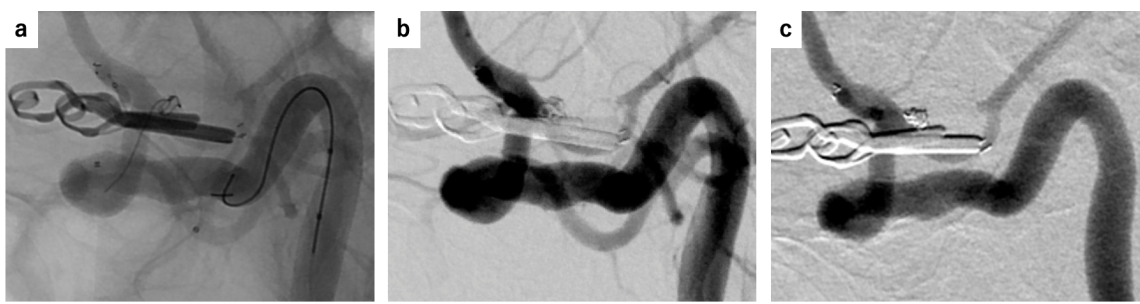

Figure 3.

\section{Hosted file}

Table.xlsx available at https://authorea.com/users/443491/articles/543480-horizontalstenting-via-retrograde-route-for-recurrent-ruptured-posterior-communicating-arteryaneurysm-after-clipping-a-case-report-and-literature-review 